\title{
Evaluation of the Association of Bariatric Surgery with Development of Cholelithiasis
}

\author{
Sultan Abdullah Bin Jerais ${ }^{1}$, Rana Mohammed Ahmad ${ }^{1}$, Mohammad Ibrhim Taleb ${ }^{2}$, Raad Jamaan \\ Al-Ghamdi ${ }^{3}$, Fahad Obaid Al-Namshah ${ }^{4}$, Sarah Mohamed Sayed ${ }^{5}$, Wejdan Abdulmoniem Alsumaien ${ }^{6}$, \\ Marram Jaber Al Haider ${ }^{6}$, Ahmed Tabaan Alenezi ${ }^{7}$, Ashwaq Musaed AL-Mutairi ${ }^{8}$ \\ 1- Al-Maarefa Colleges, 2- Sulaiman Al-Rajhi Colleges, 3- Al-Baha University, 4- King Khalid University, \\ 5-Ibn Sina National College, 6-Najran University, 7- King Faisal Specialized Hospital \& Research Center, \\ 8- Unaizah College of Medicine
}

\begin{abstract}
Background: Significant research papers have been published to assess the relation between rapid weight loss and the development of cholelithiasis, and the risk factors behind it. Assessment of cholelithiasis development after rapid weight loss as a result in most of bariatric surgery will help in reducing the admission rate to hospitals because of colic pain, and the complication related to stones formation.

Objective: This study aimed at measuring the association of rapid weight loss with the development of gallbladder stones and identifying the role of prophylactic cholecystectomy.

Methods: PubMed database was used for articles selection. All relevant articles to our review with the following topics: Bariatric Surgery, Gallbladder stones, bariatric surgery complication were included. We excluded other articles which are not related to this field. The data were extracted according to specific form in which it is going to be reviewed by group members to assess the relation between bariatric surgery and the development of stones, and weight loss as well as the benefits of prophylactic cholecystectomy against gallbladder stones.

Conclusion: Bariatric surgery can lead to gallstone formation because of the rapid excess weight loss. Small percentage may become symptomatic and rarely cholecystectomy becomes needed during the first year of the bariatric surgery. So, it is not evidently recommended to be done as prophylaxis. However, Prophylactic medicinal therapy (Ursodeoxycholic acid) in the first 6 months can be used instead.

Keywords: Bariatric Surgery, cholelithiasis, gallstone, rapid weight loss, medicinal therapy, prophylactic cholecystectomy
\end{abstract}

\section{INTRODUCTION}

The incidence of obesity has been increasing day after day, worldwide. Hence, bariatric surgery has recently become one of the most common treatment modality in maintaining long-term weight reduction and improving obesity-related conditions. There are different procedures for bariatric surgeries such as, Laparoscopic Rouxen-Y Gastric Bypass (LRYGB), Laparoscopic Sleeve Gastrectomy (LSG), and Laparoscopic Adjustable Gastric Banding (LAGB). Every procedure has its own pros and cons in relation to total weight loss, post-operative hospital stay, and the complication that might occur ${ }^{1,2}$.

Gallstones are hardened deposits of the digestive fluid bile that can form within the gallbladder. They vary in size and shape from as small as a grain of sand to as large as a golf ball. Gallstones occur when there is an imbalance in the chemical constituents of bile that result in precipitation of one or more of the components. Gallstone formation itself depends on several risk factors. In the general population, several risk factors for gallstone formation are known to increase with age with a cut-off of 40 years, female gender, obesity, and rapid weight loss as the most important contributors ${ }^{1-5}$.

Gallstones and bariatric surgery is an important issue because both obesity and bariatric surgery resulting with rapid weight loss are risk factors for the formation of gallstones ${ }^{6-8}$. The incidence of cholelithiasis after Roux-en-Y gastric bypass is reported to be as high as $71 \%^{7-9}$. Because of this high rate, some surgeons suggest a prophylactic routine cholecystectomy at the time of laparoscopic Rouxen-Y gastric bypass (LRYGB) ${ }^{\mathbf{1 0}}$. Also, some prefer performing concomitant cholecystectomy only with patients with positive preoperative ultrasound findings ${ }^{10-16}$. The percentage mentioned included both symptomatic and a symptomatic cholelithiasis. Few researchers stated that the asymptomatic gallstones in the bariatric patient can be effectively managed similar to non-obese population. 
In this paper, we are going to review the articles related to the incidence of cholelithiasis development after bariatric surgeries, and the related risk factors, and to assess the benefit of prophylaxis measures.

\section{MATERIALS AND METHODS}

\section{Sample:}

PubMed was chosen as the search database for the articles selection, because it is one of the major research databases within the suite of resources that have been developed by the National Center for Biotechnology Information (NCBI). The following keys used for the Mesh ("Bariatric Surgery/adverse effects"[Mesh] OR "Bariatric Surgery/complications"[Mesh]) AND ("Gallstones/epidemiology"[Mesh] OR "Gallstones/etiology"[Mesh] OR "Gallstones/mortality"[Mesh] OR "Gallstones/physiology"[Mesh] OR "Gallstones/prevention and control"[Mesh]). A total of 21 articles were found. Further modification by using the filter "restriction to last 5 years", which left us with 13 articles. Further screening by title and reviewing the abstracts yielded 6 articles which were enrolled. Inclusion criteria: the selected articles were based on the relevance to the project which should include one of the following topics \{Bariatric Surgery, cholelithiasis, gallbladder stones, cholecystitis \}. Exclusion criteria: all other articles which did not have one of these topics as their primary end, or repeated studies.

\section{Analysis}

No software was used, The data were extracted based on specific form that contain (Title of the study, name of the author, Objective, Summary, Results, and Outcomes), these data were reviewed by the group members to assess the relation of cholelithiasis to bariatric surgery, and weigh the pros and cons of prophylaxis measurement use. Double revision of each member's outcomes was applied to ensure the validity and minimize the mistakes.

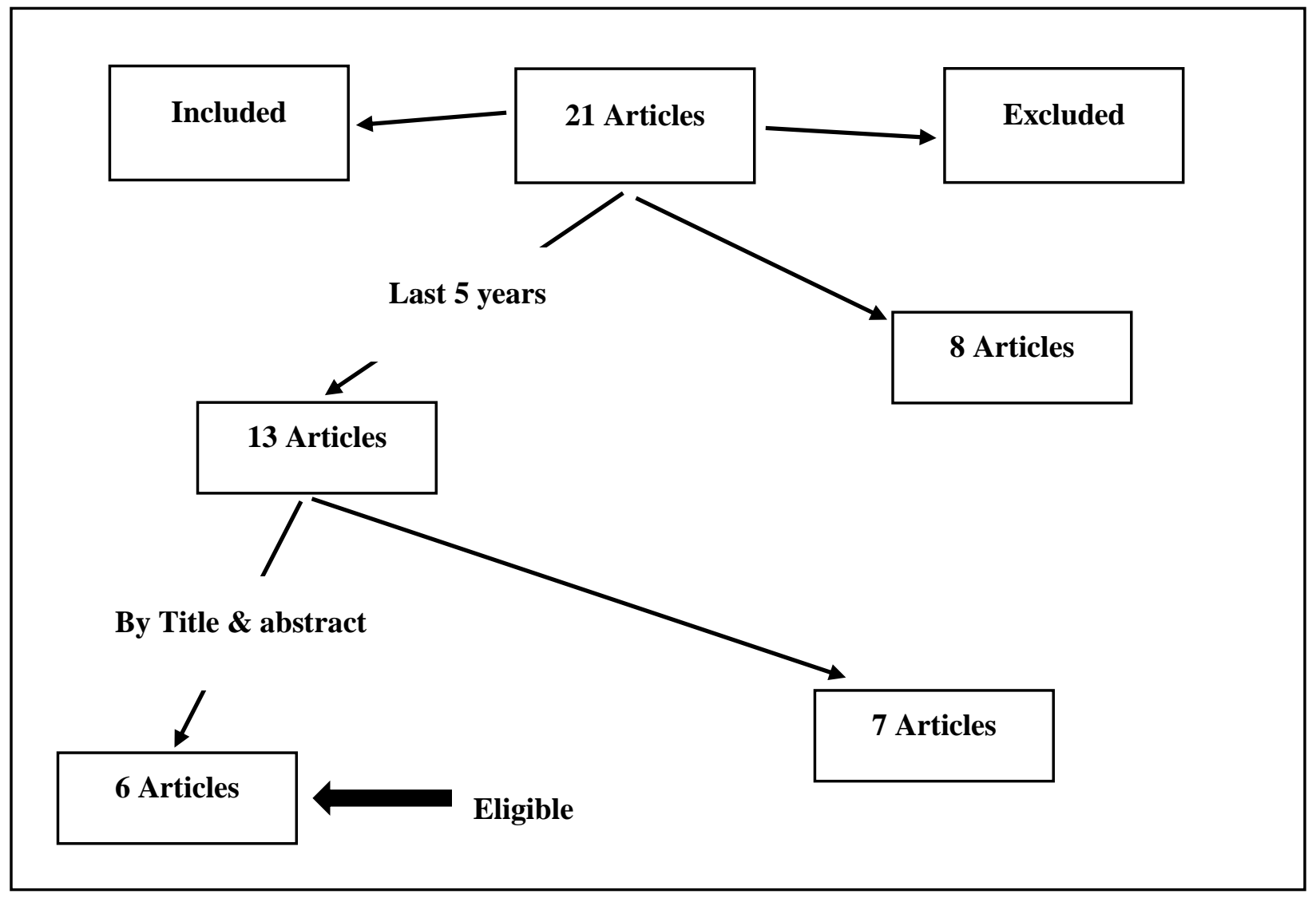

Figure 1 Articles selection 


\section{RESULTS}

\section{Study 1}

In this study the authors evaluated symptomatic cholelithiasis cases requiring cholecystectomy after each bariatric surgery procedure. A retrospective review was done between January 2009 and August 2011 , a total of 586 patients were enrolled according to specific inclusion/ exclusion criteria, 367 patients went for LRYGB, 115 went for LSG, and 104 went for LAGB.

The authors found that out of 367 LRYGB patients, $5.7 \%$ had symptomatic gallstones, 115 LSG patients, $6.1 \%$ required cholecystectomy, and of 104 LAGB patients $0 \%$ developed symptomatic gallstones. The differences in the occurrences of symptomatic gallstones between LRYGB and LSG were not statistically significant. However, statistical significance was present between LRYGB and LAGB, as well as between LSG and LAGB. Mean percentage of excess weight loss (\%EWL) at 24 months was $85.7 \%, 58.8 \%$, and $38.3 \%$ in LRYGB, LSG, and LAGB patients, respectively.

The authors explained the difference of symptomatic gallbladder stones development between different bariatric surgeries procedures by the percentage of weight loss and the time required to attain this objective .

Study 2

In this study the authors investigated the longterm effect of bariatric surgery on gallstone formation and the frequency of cholecystectomy (CHE) as well as to identifying the associated risk factors in 10 years follow up period. A total of 94 patients enrolled in the study according to specific inclusion \& exclusion criteria. Of these, 80 patients underwent Laparoscopic adjustable gastric banding (LAGB), and 14 patients had bariatric procedures other than LAGB (Gastric Bypass, and Gastric Sleeve). Mean body weight loss was $17.4 \pm 34.2 \mathrm{~kg}$ $(15 \%)$ in the LAGB group and $21.2 \pm 31.9 \mathrm{~kg}(16 \%)$ in the GB/GS-group.

The authors found that 19 patient developed gallstones, of these, 13 gallstone formations $(16.3 \%)$ occurred in the LAGB group, and $6(42.9 \%)$ in the GB/GS group.

12 female patients had cholecystectomy (CHE) after bariatric surgery due to symptomatic gallstone formation. Of these, $6 \mathrm{CHE}(8.6 \%)$ occurred in the LAGB group, and 6 (40\%) in the GB/GS-group.

The authors finalized the case study that female gender and rapid weight loss were major risk factors for postoperative cholelithiasis, and recommended for ultrasound examinations within 2 to 5 years for every patient, independent of bariatric procedure. Also they advised for a pharmacological treatment in high risk patients.

\section{Study 3}

This study was a retrospective observational study which aimed to assess the incidence of cholecystectomy after laparoscopic sleeve gastrectomy (LSG) during a 1-year follow-up and to evaluate potential risk factors and potential prophylactic measures.

A total of 361 patients who underwent primary LSG, were followed according to specific inclusion and exclusion criteria, Twenty-four (7.5\%) had symptomatic gallstones and underwent cholecystectomy after LSG.

The authors finalized that there was no significant difference between the risk of cholelithiasis development after LSG, and the general population, which does not warrant prophylactic cholecystectomy or routine pre- or postoperative ultrasound.

\section{Study 4}

This was a retrospective cohort study in which the authors evaluated the incidence of gallstone formation and symptomatic gallstones after Sleeve Gastroectomy (SG), and evaluated the association between weight loss parameters and gallstone development in these patients.

A total of 96 patients enrolled in the study according to specific inclusion, and exclusion criteria. The incidence of gallstones formation was $47.9 \%$, and the incidence of symptomatic gallstones was $22.9 \%$. The authors attributed the stones formation to the rapid weight loss from SG.

\section{Study 5}

This was a prospective study aimed at assessing the initial gallbladder status for patients going for bariatric surgery as (no disease; symptomatic or asymptomatic sludge; symptomatic or asymptomatic cholelithiasis) and convey a conservative management for those without disease and asymptomatic sludge/cholelithiasis.

Between January 2013 and January 2015, a total of 202 bariatric surgeries (184 gastric bypass and 18 sleeve gastrectomies) were performed.

According to specific inclusion and exclusion criteria, a total of 146 patients were enrolled. During 12 month follow up period de novo gallbladder disease was observed in $21.2 \%$. The overall rate of cholecystectomy because of symptomatic disease was $3.4 \%$. 
Evaluation of the Association of Bariatric Surgery...

The authors finalized that Conservative management of asymptomatic gallbladder disease in candidates to bariatric surgery is safe and can be offered in every case, based on the low percentage of patients requiring further cholecystectomy after 12 months. Also, a conservative management can be offered to patients developing de novo sludge/cholelithiasis without related symptoms.

\section{Study 6}

The authors of this study assessed time needed to carry out cholecystectomy after bariatric surgery, and identifying predisposing factors for the development of gallbladder disease after bariatric surgery.

Data prospectively collected for patient going for different bariatric surgery procedures, (laparoscopic Roux-en-Y gastric bypass (LRYGB), laparoscopic sleeve gastrectomy (LSG), and laparoscopic adjustable gastric banding (LAGB)).

A total of 1398 patients were enrolled according to specific inclusion and exclusion criteria. The study showed that bariatric surgery was associated with low rate $(7.8 \%)$ post-operative cholecystectomy within 4 years and with a higher rate after RYGB than after LAGB or LSG. The rates were highest in the first 6 months after surgery and decline overtime. The excess weight loss (EWL) $>25 \%$ within the first 3 months was the strongest predictor of postoperative cholecystectomy. In addition, white patients had 1.45 times higher cholecystectomy rates than did black patients. Preoperative body mass index, gender, and surgeon did not affect cholecystectomy rates.

\section{DISCUSSION}

Looking at the included studies, we found that the main factor behind developing gallstones after bariatric surgery is mostly isolated to rapid excess weight loss. Most of the studies implied that the relationship is proportional between gallstones formation post-operatively and how fast losing weight was either in obese and non-obese patients. According to some of the studies, developing gallbladder sludge or stone as a complication of bariatric surgery can be managed conservatively ${ }^{19-22}$. Low percentage only required further surgical intervention (lap-chole) because the stones rarely become symptomatic. Some of the studies (like study 1 and 2) made a comparison among the different types of bariatric surgery (laparoscopic Roux-en-Y gastric bypass (LRYGB), laparoscopic sleeve gastrectomy (LSG), and laparoscopic adjustable gastric banding (LAGB)) regarding the complications. They found that LAGB is the least one to lead to symptomatic gallstone and it is slower in weight reduction after it more than after LRYGB and $\mathrm{LSG}^{18-19-23}$. So, they related this result to how rapid and how much the excess of weight loss will be post operatively. In addition, some studies found that the use of Ursodeoxycholic acid as a prophylactic medicinal therapy after bariatric surgery is very helpful in preventing gallstone formation specifically during the rapid excess weight loss ${ }^{19}$. So, the idea of making prophylactic cholecystectomy a part of every bariatric surgery is not evidently proved nor well supported. We suggest according to the studies that abdominal ultrasound should be done for every patient who develops any abdominal pain during the follow up at least within the first 12 months postoperatively ${ }^{19-20}$.

\section{Strengths and Limitations}

Our final review was on 6 major studies. Unfortunately, it is not a high number of studies. However, the collected data were sufficient and enough to provide us with good results. We tried to choose the similar ones to each other in the methods and approach especially the studies with the same aim, the one we wanted. So, we only used the search engine of PubMed. Therefore, we may missed other major studies relevant to our review holding additional data that may widen our gaze while analyzing and comparing the results. During the selection, we double-reviewed the studies and we made sure that the studies we included are fit to be considered as major study, despite some of them were finalized upon small sample size which might provide bias to the review. In addition, we used the filter of the search engine to limit the result to the last 5 years only.

\section{Future perspective}

In some studies, cholelithiasis after LAGB was found to be less common than other surgeries ${ }^{18-19-23}$. So, it will be good to approach this aspect with a study regarding which type of bariatric surgery should be favored and recommended over the others. A few number of studies mentioned about Ursodeoxycholic acid and its effect on the gallstone formation prevention. So, we advise to conduct more studies about the use of it and its effect after bariatric surgeries. Making post-operative ultrasound during the first year a part of the follow up is still controversial. Conducting a study to weigh the benefits of post-operative ultrasound over the cost and trouble in order to prevent or to predict the next step of management might be useful. 


\section{CONCLUSION}

Rapid excess weight loss is found to be the main factor behind gallstone formation after Bariatric surgery. During the first year after the bariatric surgery, small percentage of patients may become symptomatic and rarely cholecystectomy becomes needed. So, it is not evidently recommended to be done as prophylaxis. However, Prophylactic medicinal therapy (Ursodeoxycholic acid) in the first 6 months can be used instead.

\section{REFERENCES}

1. Maurer KR, Everhart JE, Ezzati TM et al.(1989): Prevalence of gallstone disease in Hispanic populations in the United States. Gastroenterology, 96(2 Pt 1):487-92.

2. Barbara L, Sama C, MorselliLabate A et al.(1987): A population study on the prevalence of gallstone disease: the Sirmione Study. Hepatology, 7(5):913-7.

3. Attili AF, Carulli $\mathbf{N}$, Roda $\mathbf{E}$ et al.(1995): Epidemiology of gallstone disease in Italy: prevalence data of the Multicenter Italian Study on Cholelithiasis (M.I.COL.). Am J Epidemiol., 141(2):158-65

4. Mabee TM, Meyer P, DenBesten L et al. (1979): The mechanism of increased gallstone formation in obese human subjects. Surgery, 79(4):460-8.

5. Shiffman ML, Sugerman HJ, Kellum JM et al. (1992): Moore EW. Changes in gallbladder bile composition following gallstone formation and weight reduction. Gastroenterology, 103(1):214-21

6. Brandao L, Oliveira C, AdamiChaim E et al. (2003): Impact of rapid weight reduction on risk of cholelithiasis after bariatric surgery. Obes Surg.,13:6258.

7. Dittrick GW, Thompson JS, Campos D et al. (2005): Gallbladder pathology in morbid obesity. Obes Surg., 15:238-42.

8. Papavramidis S, Deligianidis $\mathbf{N}$, Papavramidis $\mathbf{T}$ et al. (2003):Laparoscopic cholecystectomy after bariatric surgery. SurgEndosc., 17:1061-4

9. Nagem $R, \quad$ Lazaro-da-Silva A (2012):Cholecystolithiasis after gastric bypass: a clinical, biochemical, and ultrasonographic 3-year follow-up study. Obes Surg., 22:1594-9.

10. Bastouly M, Arasaki CH, Ferreira JB et al. (2009): Early changes in postprandial gallbladder emptying in morbidly obese patients undergoing Rouxen-Y gastric bypass: correlation with the occurrence of biliary sludge and gallstones. ObesSurg .,9:22-8.

11. Teivelis MP, Faintuch J, Ishida $R$ et al. (2007): Endoscopic and ultrasonographic evaluation before and after Roux-en-Y gastric bypass for morbid obesity. Arq Gastroenterol., 44:8-13.

12. Villegas L, Schneider B, Provost D et al.(2004): Is routine cholecystectomy required during laparoscopic gastric bypass? ObesSurg., 14: 206-11.

13. Tarantino I, Warschkow R, Steffen T et al. (2011): Is routine cholecystectomy justified in severely obese patients undergoing a laparoscopic Roux-en-Y gastric bypass procedure? A comparative cohort study. Obes Surg., 21:1870-8.

14. Nougou A, Suter M (2008): Almost routine prophylactic cholecystectomy during laparoscopic gastric bypass is safe. Obes Surg.,18:535-9.

15. Tucker ON, Fajnwaks $P$, Szomstein $S$ et al. (2008):Is concomitant cholecystectomy necessary in obese patients undergoing laparoscopic gastric bypass surgery? SurgEndosc., 22:2450-4.

16. Hamad GG, Ikramuddin S, Gourash WF et al. (2004):Elective cholecystectomy during laparoscopic Roux-en-Y gastric bypass: is it worth the wait? Obes Surg., 13:76-81

17. Swartz DE, Felix EL (2005): Elective cholecystectomy after Roux-en-Y gastric bypass: why should asymptomatic gallstones be treated differently in morbidly obese patients? SurgObes Relat Dis.,1: 55560.

18. Rena $\mathrm{C} M$, Teixeira AF, Ducoin $\mathrm{C}$ et al. (2014):Comparison of Cholecystectomy Cases after Roux-en-Y Gastric Bypass, Sleeve Gastrectomy, and Gastric Banding. SurgObes Relat Dis., 10(1): 64-68.

19. Andreas M, Sturm W, Kuhnert B et al. (2015): Incidence of Gallstone Formation and Cholecystectomy 10 Years After Bariatric Surgery. Obesity Surgery, 25(7): 1171-176.

20. Dakour A, Sultanem S, Abtar $\mathrm{H}$ et al. (2016): Management of Gallbladder Disease after Sleeve Gastrectomy in a Selected Lebanese Population. SurgObes Relat Dis., 12(7): 1300-304.

21. Wuttiporn W, Leelasinjaroen $\mathrm{P}$, Al-Hamid $\mathrm{H}$ et al. (2016): The Incidence of Cholelithiasis after Sleeve Gastrectomy and Its Association with Weight Loss: A Two-centre Retrospective Cohort Study. International Journal of Surgery, 30: 13-18.

22. Omar P, Hernán G, Amado et al. (2016): A Prospective Study of the Conservative Management of Asymptomatic Preoperative and Postoperative Gallbladder Disease in Bariatric Surgery. Obesity Surgery, 27(1):148-53.

23. Tsirline V, Keilani Z, El Djouzi S et al. (2014): How Frequently and When Do Patients Undergo Cholecystectomy after Bariatric Surgery?" SurgObes Relat Dis., 10(2):313-21. 